\title{
Adaptive exponential lag synchronization for neural networks with mixed delays via intermittent control
}

\author{
Peipei Zhou and Shuiming Cai
}

"Correspondence:

caishuiming2008@126.com

Faculty of Science, Jiangsu

University, Zhenjiang, China

\section{Springer}

\begin{abstract}
In this paper, the problem of exponential lag synchronization for a class of neural networks with mixed delays including discrete and distributed delays is investigated via adaptive intermittent control. Based on piecewise analytic method, some sufficient conditions for globally exponential lag synchronization are established through constructing a piecewise continuous auxiliary function. It is noted that both the control periods and the control widths in our adaptive intermittent control strategy are allowed to be nonidentical, which extends the scope of application of periodically intermittent control with fixed both control period and control width employed widely in previous works. Moreover, it is shown that the derived globally exponential lag synchronization criteria are related to the control rates rather than the control periods, which facilitates the choice of the control periods for practical problems. Finally, a numerical example is given to illustrate the correctness of the obtained theoretical results.
\end{abstract}

Keywords: exponential lag synchronization; neural networks; mixed delays; nonperiodically intermittent control; adaptive strategy

\section{Introduction}

Since the pioneering work of Pecora and Carroll [1], synchronization of chaotic systems has attracted much attention from researchers in various fields because of its potential applications in secure communication, signal processing, biological systems and so on [2, 3]. Up to now, several different kinds of synchronous patterns have been discovered and deeply studied, such as complete synchronization [1], generalized synchronization [4], phase synchronization [5], lag synchronization [6] and projective synchronization [7].

Lag synchronization, defined as the state of the drive system is delayed by a positive constant in comparison with that of the response system, is an interesting phenomenon and has been observed in neural models, electronic circuits and lasers [3, 8-11]. In many practical situations, due to the finite transmission speed of signals, it is more reasonable to require the response system to synchronize with the drive system at a time lag rather than at exactly the same time [12-15]. For example, in a telephone communication system, the voice one hears on the receiver side at time $t$ is the voice from the transmitter side at time $t-\tau[12,13]$. Therefore, how to effectively make two chaotic systems achieve lag synchronization is an important issue which deserves detailed investigation. Additionally,

(c) The Author(s) 2018. This article is distributed under the terms of the Creative Commons Attribution 4.0 International License (http://creativecommons.org/licenses/by/4.0/), which permits unrestricted use, distribution, and reproduction in any medium, provided you give appropriate credit to the original author(s) and the source, provide a link to the Creative Commons license, and indicate if changes were made. 
in implementation of neural networks, time delays always exist in the signal transmission among neurons owing to the finite switching speed of neurons and amplifiers, which will affect the dynamical behaviors of neural networks [13, 16-18]. Hence, time delays should be taken into account when exploring the dynamics of neural networks. In the light of these facts, many efforts have been made devoted to the study of lag synchronization of delayed chaotic systems and delayed neural networks in recent years [11-15, 19-23]. For instance, the problem of lag synchronization control for memristor-based coupled delayed neural networks with parameter mismatches was explored in [11]. In [12], the effect of parameter mismatch on lag synchronization of coupled delayed systems was investigated by periodically intermittent control. In [13], the exponential lag synchronization for neural networks with mixed delays was considered via periodically intermittent control. In [21], the lag synchronization for competitive neural networks with mixed delays and uncertain hybrid perturbations was studied via adaptive control. In [23], the adaptive lag synchronization of Cohen-Grossberg neural networks with discrete delays was discussed.

In order to guarantee synchronization between two chaotic systems can be realized, several control schemes have been proposed, such as feedback control, adaptive control, intermittent control and impulsive control [12-15, 19-32]. Intermittent control, as a discontinuous control method, has been adopted in engineering fields in view of its convenient implementation in engineering control [33-38]. Recently, an intermittent control scheme with fixed both control period and control width, namely periodically intermittent control scheme, has been developed to study the synchronization problem for chaotic systems as well as dynamical networks; see [12, 13, 28-43] and references cited therein. However, in practical applications, the requirement that both control period and control width be fixed is evidently unreasonable. Actually, a more reasonable intermittent control is nonperiodic (or aperiodic), where both control period and control width are allowed to be variable $[44,45]$. Obviously, nonperiodically intermittent control is more feasible than the periodically intermittent one, as the latter can be regarded as a special case of the former. Therefore, it is necessary to investigate the synchronization problem under nonperiodically intermittent control. Presently, some initial work has been done on this topic [44-53]. In [44-46], the synchronization of delayed dynamical networks via nonperiodically intermittent pinning control was studied. In [47], the adaptive synchronization problem for neural networks with stochastic perturbation under nonperiodically intermittent control was considered. In [48], the authors investigated the synchronization of chaotic systems with mixed delays by using nonperiodically intermittent control strategy. In [49, 50], the authors discussed the problem of adaptive outer synchronization between two delayed dynamical networks via nonperiodically intermittent pinning control. In [52], the aperiodically intermittent synchronization for directed dynamical networks with switching topologies was investigated.

As is well known, in real applications, obtaining the identical parameters of the drive and response systems directly is impossible. For such case, adaptive approach can be applied to deal with the synchronization problem [14, 21, 23]. Moreover, in biological neural networks, it often has a spatial extent because of the presence of parallel pathways with a variety of axon sizes and lengths [54-56]. Therefore, there exists a distribution of transmission delay, which is not suitable to be modeled with discrete delay. A more appropriate approach is to incorporate the distributed delay in the neural networks model. However, to the best of our knowledge, there are few studies concerned with the lag synchronization 
problem for neural networks with distributed delay via adaptive nonperiodically intermittent control.

Motivated by the above discussion, this paper aims to study the problem of exponential lag synchronization for a class of neural networks with both discrete and distributed delays via adaptive nonperiodically intermittent control. By introducing a piecewise continuous auxiliary function, some sufficient conditions for globally exponential lag synchronization are established by virtue of piecewise analytic method. A numerical example is finally provided to demonstrate the effectiveness of the proposed control methodology. The main contributions of this paper can be outlined as follows: (1) different from the periodically intermittent control adopted in previous works [12, 13, 28-43], here the adaptive intermittent control is nonperiodic with non-fixed both control period and control width, which extends the intermittent control strategy's application scope; (2) unlike in the references [39-41,50], the candidate Lyapunov function introduced in this paper is piecewise continuous, and then by means of which, some criteria ensuring globally exponential lag synchronization are established; (3) the derived theoretical results indicate that the lag synchronization criteria are related to the control rates rather than the control periods, which is propitious to selecting the control periods in practical applications.

\section{Model description and preliminaries}

Consider a class of neural networks with both discrete and distributed delays given by

$$
\begin{aligned}
\dot{x}_{i}(t)= & -c_{i}\left(x_{i}(t)\right)+\sum_{j=1}^{n} a_{i j} f_{j}\left(x_{j}(t)\right)+\sum_{j=1}^{n} b_{i j} g_{j}\left(x_{j}\left(t-\tau_{j}\right)\right) \\
& +\sum_{j=1}^{n} d_{i j} \int_{t-\sigma_{j}}^{t} h_{j}\left(x_{j}(s)\right) \mathrm{d} s+I_{i}, \quad i \in \mathfrak{T}=\{1,2, \ldots, n\},
\end{aligned}
$$

where $x_{i}(t)$ denotes the state of the $i$ th neuron at time $t, f_{j}(\cdot), g_{j}(\cdot)$ and $h_{j}(\cdot)$ are the neuron activation functions of the $j$ th neuron. The constants $a_{i j}, b_{i j}$ and $d_{i j}$ are the connection weight, the discrete-delay connection weight and the distributed-delay connection weight of the $j$ th neuron on the $i$ th neuron, respectively. $I_{i}$ is an external input, $c_{i}(\cdot)$ corresponds to the rate with which the potential of the $i$ th neuron will be reset to the resting state in isolation [54]. $\tau_{j} \geq 0$ is the transmission delay, while $\sigma_{j} \geq 0$ describes the distributed delay. The initial condition of neural networks (1) is given by $x_{i}(v)=\varphi_{i}(v),-\eta \leq v \leq 0, i \in \mathfrak{T}$, where $\eta=\max \{\tau, \sigma\}, \tau=\max _{j \in \mathfrak{T}}\left\{\tau_{j}\right\}, \sigma=\max _{j \in \mathfrak{T}}\left\{\sigma_{j}\right\}, \varphi(v)=\left(\varphi_{1}(v), \varphi_{2}(v), \ldots, \varphi_{n}(v)\right)^{\top} \in$ $\mathcal{C}\left([-\eta, 0], \mathbb{R}^{n}\right)$, and $\mathcal{C}\left([-\eta, 0], \mathbb{R}^{n}\right)$ denotes the Banach space of all continuous functions mapping $[-\eta, 0]$ into $\mathbb{R}^{n}$ with norm defined by $\|\varphi\|=\sup _{v \in[-\eta, 0]}\left\{\max _{j \in \mathfrak{T}}\left|\phi_{j}(v)\right|\right\}$.

To proceed, we make the following assumptions for system (1).

Assumption 1 For each $i \in \mathfrak{T}$, function $c_{i}(\cdot): \mathbb{R} \rightarrow(0,+\infty)$ is continuous and monotone increasing, i.e., there exists a positive constant $C_{i}>0$ such that

$$
\frac{c_{i}\left(s_{1}\right)-c_{i}\left(s_{2}\right)}{s_{1}-s_{2}} \geq C_{i}
$$

for all $s_{1}, s_{2} \in \mathbb{R}$ and $s_{1} \neq s_{2}$. 
Assumption 2 For each $j \in \mathfrak{T}$, functions $f_{j}(\cdot), g_{j}(\cdot)$ and $h_{j}(\cdot)$ are Lipschitz continuous, i.e., there exist positive constants $F_{j}>0, G_{j}>0$ and $H_{j}>0$ such that

$$
\begin{aligned}
& \left|f_{j}\left(s_{1}\right)-f_{j}\left(s_{2}\right)\right| \leq F_{j}\left|s_{1}-s_{2}\right|, \quad\left|g_{j}\left(s_{1}\right)-g_{j}\left(s_{2}\right)\right| \leq G_{j}\left|s_{1}-s_{2}\right|, \\
& \left|h_{j}\left(s_{1}\right)-h_{j}\left(s_{2}\right)\right| \leq H_{j}\left|s_{1}-s_{2}\right|,
\end{aligned}
$$

for all $s_{1}, s_{2} \in \mathbb{R}$.

In this paper, we aim to drive system (1) to achieve lag synchronization via adaptive intermittent control in drive-response configuration. Therefore, we regard system (1) as the drive system and design the following response system:

$$
\begin{aligned}
\dot{y}_{i}(t)= & -c_{i}\left(y_{i}(t)\right)+\sum_{j=1}^{n} a_{i j} f_{j}\left(y_{j}(t)\right)+\sum_{j=1}^{n} b_{i j} g_{j}\left(y_{j}\left(t-\tau_{j}\right)\right) \\
& +\sum_{j=1}^{n} d_{i j} \int_{t-\sigma_{j}}^{t} h_{j}\left(y_{j}(s)\right) \mathrm{d} s+I_{i}+u_{i}(t), \quad i \in \mathfrak{T},
\end{aligned}
$$

where $y_{i}(t)$ represents the state of the $i$ th neuron at time $t$, the initial values of the response system (2) are $y_{i}(v)=\psi_{i}(v),-\eta \leq v \leq 0, i \in \mathfrak{T}$ and $\psi(v)=\left(\psi_{1}(v), \psi_{2}(v), \ldots, \psi_{n}(v)\right)^{\top} \in$ $\mathcal{C}\left([-\eta, 0], \mathbb{R}^{n}\right), u_{i}(t)$ is an adaptive nonperiodical intermittent controller described by

$$
u_{i}(t)=-k_{i}(t)\left(y_{i}(t)-x_{i}(t-\omega)\right)
$$

in which $\omega \geq 0$ is propagation delay between the output of the response system and that of the drive system, and $k_{i}(t)$ is the adaptive intermittent feedback control gain given by

$$
k_{i}(t)= \begin{cases}k_{i}(0), & t=0, \\ k_{i}\left(t_{l}+\delta_{l}\right), & t=t_{l+1}, \\ 0, & t_{l}+\delta_{l}<t<t_{l+1},\end{cases}
$$

with the updating law

$$
\dot{k}_{i}(t)=h_{i} \exp \left\{\rho_{0} t\right\}\left(y_{i}(t)-x_{i}(t-\omega)\right)^{2}, \quad t_{l} \leq t \leq t_{l}+\delta_{l},
$$

where $m \in \mathbb{N}^{+}=\{1,2, \ldots\}, \rho_{0}>0$ is a positive constant, $h_{i}>0$ and $k_{i}(0)>0$ for $i \in \mathfrak{T}$. The time sequence $\left\{t_{l}\right\}_{l=1}^{+\infty}$ satisfies $0=t_{1}<t_{2}<\cdots<t_{l}<\cdots$ and $\lim _{l \rightarrow+\infty} t_{l}=+\infty$. The time span $\left[t_{l}, t_{l+1}\right)$ is the time of the $l$ th period, and $\left(t_{l+1}-t_{l}\right)$ is referred to as the $l$ th control period; $\left[t_{l}, t_{l}+\delta_{l}\right]$ is the $l$ th work time, and $\delta_{l}>0$ is referred to as the $l$ th control width (control duration); $\left(t_{l}+\delta_{l}, t_{l+1}\right)$ is the $l$ th rest time, and $\left(t_{l+1}-t_{l}\right)-\delta_{l}>0$ is referred to as the $l$ th rest width (rest duration).

Remark 1 Note that controller (3) is a kind of nonperiodical control, and each control period is composed of work time and rest time. During the work time $\left(t_{l} \leq t \leq t_{l}+\delta_{l}\right)$, the controller is performed according to the adaptive update law (5), while it is removed during the rest time $\left(t_{l}+\delta_{l}<t<t_{l+1}\right)$. It should be stressed that here the control period 
$\left(t_{l+1}-t_{l}\right)$ and control width $\delta_{l}$ are both non-fixed, and hence this control strategy is more general. Obviously, when $t_{l+1}-t_{l} \equiv \mathrm{T}$ and $\delta_{l} \equiv \delta, l \in \mathbb{N}^{+}$, the adaptive intermittent control type becomes the periodic one, which has been studied in [39-41].

For convenience of analysis, let $\mathrm{T}_{0}=\overline{\mathrm{T}}_{0}=t_{1}, \mathrm{~T}_{l}=t_{l+1}-t_{l}, \overline{\mathrm{T}}_{l}=\sum_{j=0}^{l} \mathrm{~T}_{j}$, and $\theta_{l}=\delta_{l} / \mathrm{T}_{l}$, $l \in \mathbb{N}^{+}$, where $\theta_{l}$ is called the $l$ th control rate. Then we get $t_{l}=\overline{\mathrm{T}}_{l-1}$ and $\delta_{l}=\theta_{l} \mathrm{~T}_{l}, l \in \mathbb{N}^{+}$. Suppose that $x(t)=\left(x_{1}(t), x_{2}(t), \ldots, x_{n}(t)\right)^{\top}$ and $y(t)=\left(y_{1}(t), y_{2}(t), \ldots, y_{n}(t)\right)^{\top}$ are solutions of systems (1) and (2) with different initial conditions, respectively. Denote $z_{i}(t)=y_{i}(t)-$ $x_{i}(t-\omega)$ be the synchronous error between the states of the drive system (1) and response system (2), then we can obtain the following error system:

$$
\left\{\begin{aligned}
\dot{z}_{i}(t)= & -\tilde{c}_{i}\left(z_{i}(t)\right)+\sum_{j=1}^{n} a_{i j} \tilde{f}_{j}\left(z_{j}(t)\right)+\sum_{j=1}^{n} b_{i j} \tilde{g}_{j}\left(z_{j}\left(t-\tau_{j}\right)\right) \\
& +\sum_{j=1}^{n} d_{i j} \int_{t-\sigma_{j}}^{t} \tilde{h}_{j}\left(z_{j}(s)\right) \mathrm{d} s-k_{i}(t) z_{i}(t), \quad \overline{\mathrm{T}}_{l-1} \leq t \leq \overline{\mathrm{T}}_{l-1}+\theta_{l} \mathrm{~T}_{l}, \\
\dot{z}_{i}(t)= & -\tilde{c}_{i}\left(z_{i}(t)\right)+\sum_{j=1}^{n} a_{i j} \tilde{f}_{j}\left(z_{j}(t)\right)+\sum_{j=1}^{n} b_{i j} \tilde{g}_{j}\left(z_{j}\left(t-\tau_{j}\right)\right) \\
& +\sum_{j=1}^{n} d_{i j} \int_{t-\sigma_{j}}^{t} \tilde{h}_{j}\left(z_{j}(s)\right) \mathrm{d} s, \quad \overline{\mathrm{T}}_{l-1}+\theta_{l} \mathrm{~T}_{l}<t<\overline{\mathrm{T}}_{l},
\end{aligned}\right.
$$

where $i \in \mathfrak{T}, l \in \mathbb{N}^{+}, \tilde{c}_{i}\left(z_{i}(t)\right)=c_{i}\left(y_{i}(t)\right)-c_{i}\left(x_{i}(t-\omega)\right), \tilde{f}_{j}\left(z_{j}(t)\right)=f_{j}\left(y_{j}(t)\right)-f_{j}\left(x_{j}(t-\omega)\right), \tilde{g}_{j}\left(z_{j}(t-\right.$ $\left.\left.\tau_{j}\right)\right)=g_{j}\left(y_{j}\left(t-\tau_{j}\right)\right)-g_{j}\left(x_{j}\left(t-\tau_{j}-\omega\right)\right)$, and $\tilde{h}_{j}\left(z_{j}(s)\right)=h_{j}\left(y_{j}(s)\right)-h_{j}\left(x_{j}(s-\omega)\right)$.

In order to define the initial condition of system (6), we supplement the state $x_{i}(t)$ on $[-\eta-\omega,-\eta]$ as $x_{i}(v)=\varphi_{i}(-\eta),-\eta-\omega \leq v \leq-\eta, i \in \mathfrak{T}$. For $i \in \mathfrak{T}$, through introducing the notation $\bar{\varphi}_{i}(v)$ with

$$
\bar{\varphi}_{i}(v)= \begin{cases}\varphi_{i}(v), & -\eta \leq v \leq 0, \\ \varphi_{i}(-\eta), & -\eta-\omega \leq v \leq-\eta,\end{cases}
$$

then the initial condition of system (6) is given by $z_{i}(v)=\psi_{i}(v)-\bar{\varphi}_{i}(v-\omega),-\eta \leq v \leq 0$, $i \in \mathfrak{T}$.

Definition 1 The drive system (1) and response system (2) are said to be globally exponentially lag synchronized, if for any initial values $\psi(s), \varphi(s) \in \mathcal{C}\left([-\eta, 0], \mathbb{R}^{n}\right)$, there exist positive constants $M>0$ and $\varepsilon>0$ such that

$$
\|y(t)-x(t-\omega)\| \leq M \exp \{-\varepsilon t\}, \quad t \geq 0
$$

If $\omega=0$, the synchronization is complete synchronization.

\section{Main results}

In this section, by the adaptive nonperiodical intermittent controllers (3)-(5), we consider the globally exponential lag synchronization between the drive-response systems (1) and (2). Some criteria ensuring the globally exponential lag synchronization will be established by means of piecewise analytic method. For convenience, we introduce the following notations.

For $\kappa \in \mathfrak{T}$, let $\alpha_{\kappa}=-2 C_{\kappa}+F_{\kappa}\left|a_{\kappa \kappa}\right|+\sum_{j=1}^{n} F_{j}\left|a_{\kappa j}\right|+\sum_{j=1}^{n} G_{j}\left|b_{\kappa j}\right|+\sum_{j=1}^{n} \sigma_{j} H_{j}\left|d_{\kappa j}\right|$, $\alpha=\max _{\kappa \in \mathfrak{T}}\left\{\alpha_{\kappa}\right\}, \beta_{\kappa}=\sum_{j=1, j \neq \kappa}^{n} F_{j}\left|a_{\kappa j}\right|, \gamma_{\kappa}=\sum_{j=1}^{n} G_{j}\left|b_{\kappa j}\right|, \zeta_{\kappa}=\sum_{j=1}^{n} \sigma_{j} H_{j}\left|d_{\kappa j}\right|$, and $\pi_{0}=$ $\max _{\kappa \in \mathfrak{T}}\left\{\beta_{\kappa}+\gamma_{\kappa}+\zeta_{\kappa}\right\}$. 
Theorem 1 Suppose that Assumptions 1-2 hold and $\inf _{l \in \mathbb{N}^{+}}\left\{\theta_{l}\right\}=\theta_{\text {inf }}>0$, by the adaptive nonperiodical intermittent controllers (3)-(5), the globally exponential lag synchronization between the drive-response systems (1) and (2) can be achieved if there exists a positive constant $\rho_{0}>\pi_{0}$ such that

$$
\lambda-\left(1-\theta_{\text {inf }}\right)\left(\rho_{0}+\alpha^{+}\right)>0,
$$

where $\alpha^{+}=\max \{0, \alpha\}, \lambda=\min _{\kappa \in \mathfrak{T}}\left\{\lambda_{\kappa}\right\}$, and $\lambda_{\kappa}$ is the unique positive solution of the equation $\lambda_{\kappa}-\rho_{0}+\beta_{\kappa}+\gamma_{\kappa} \exp \left\{\lambda_{\kappa} \tau\right\}+\zeta_{\kappa} \exp \left\{\lambda_{\kappa} \sigma\right\}=0$.

Proof Denote $\Psi_{\kappa}\left(\epsilon_{\kappa}\right)=\epsilon_{\kappa}-\rho_{0}+\beta_{\kappa}+\gamma_{\kappa} \exp \left\{\epsilon_{\kappa} \tau\right\}+\zeta_{\kappa} \exp \left\{\epsilon_{\kappa} \sigma\right\}, \kappa \in \mathfrak{T}$. Since $\rho_{0}>\pi_{0}$, one has $\Psi_{\kappa}(0)<0, \Psi_{\kappa}(+\infty)>0$ and $\mathrm{d} \Psi_{\kappa} / \mathrm{d} \epsilon_{\kappa}>0$. By the continuity and the monotonicity of $\Psi_{\kappa}\left(\epsilon_{\kappa}\right)$, the equation $\epsilon_{\kappa}-\rho_{0}+\beta_{\kappa}+\gamma_{\kappa} \exp \left\{\epsilon_{\kappa} \tau\right\}+\zeta_{\kappa} \exp \left\{\epsilon_{\kappa} \sigma\right\}=0$ has an unique positive solution $\lambda_{\kappa}>0$. Notice that $\lambda=\min _{\kappa \in \mathfrak{T}}\left\{\lambda_{\kappa}\right\}>0$ and $\Psi_{\kappa}\left(\epsilon_{\kappa}\right)$ is increasing with respect to $\epsilon_{\kappa}$ for any $\kappa \in \mathfrak{T}$, then we get

$$
\lambda-\rho_{0}+\beta_{\kappa}+\gamma_{\kappa} \exp \{\lambda \tau\}+\zeta_{\kappa} \exp \{\lambda \sigma\} \leq 0, \quad \text { for all } \kappa \in \mathfrak{T} .
$$

Construct a piecewise function defined as follows:

$$
\Upsilon_{i}(t)= \begin{cases}\frac{1}{2} \exp \left\{-\rho_{0} t\right\} \frac{1}{h_{i}}\left(k_{i}(t)-k^{*}\right)^{2}, & \overline{\mathrm{T}}_{l-1} \leq t \leq \overline{\mathrm{T}}_{l-1}+\theta_{l} \mathrm{~T}_{l}, \\ \frac{1}{2} \exp \left\{-\rho_{0} t\right\} \frac{1}{h_{i}}\left(k_{i}\left(\overline{\mathrm{T}}_{l-1}+\theta_{l} \mathrm{~T}_{l}\right)-k^{*}\right)^{2}, & \overline{\mathrm{T}}_{l-1}+\theta_{l} \mathrm{~T}_{l}<t<\overline{\mathrm{T}}_{l},\end{cases}
$$

where $i \in \mathfrak{T}, l \in \mathbb{N}^{+}$, and $k^{*}>0$ is a positive constant to be determined later. From (4) and (9), it is easy to see that $\Upsilon_{i}(t)$ is continuous for all $t \geq 0$ and $i \in \mathfrak{T}$.

Denote $P_{i}(t)=\frac{1}{2} z_{i}^{2}(t), i \in \mathfrak{T}$ and consider the following candidate Lyapunov functions:

$$
V_{i}(t)=P_{i}(t)+\Upsilon_{i}(t), \quad i \in \mathfrak{T} .
$$

Obviously, $V_{i}(t)$ is continuous for all $t \geq 0$ and $i \in \mathfrak{T}$ because $P_{i}(t)$ is continuous for all $t \geq 0$ and $i \in \mathfrak{T}$.

When $\overline{\mathrm{T}}_{l-1} \leq t \leq \overline{\mathrm{T}}_{l-1}+\theta_{l} \mathrm{~T}_{l}, l \in \mathbb{N}^{+}$, by Assumptions 1 and 2 , the derivative of $V_{i}(t)$ with respect to time $t$ along the trajectories of system (6) can be calculated as follows:

$$
\begin{aligned}
\dot{V}_{i}(t)= & z_{i}(t)\left[-\tilde{c}_{i}\left(z_{i}(t)\right)+\sum_{j=1}^{n} a_{i j} \tilde{f}_{j}\left(z_{j}(t)\right)+\sum_{j=1}^{n} b_{i j} \tilde{g}_{j}\left(z_{j}\left(t-\tau_{j}\right)\right)\right. \\
& \left.+\sum_{j=1}^{n} d_{i j} \int_{t-\sigma_{j}}^{t} \tilde{h}_{j}\left(z_{j}(s)\right) \mathrm{d} s-k_{i}(t) z_{i}(t)\right] \\
& -\frac{\rho_{0}}{2} \exp \left\{-\rho_{0} t\right\} \frac{1}{h_{i}}\left(k_{i}(t)-k^{*}\right)^{2}+\left(k_{i}(t)-k^{*}\right) z_{i}^{2}(t) \\
\leq & -C_{i} z_{i}^{2}(t)+\left|z_{i}(t)\right|\left[\sum_{j=1}^{n}\left|a_{i j}\right| F_{j}\left|z_{j}(t)\right|+\sum_{j=1}^{n}\left|b_{i j}\right| G_{j}\left|z_{j}\left(t-\tau_{j}\right)\right|\right. \\
& \left.+\sum_{j=1}^{n}\left|d_{i j}\right| H_{j} \int_{t-\sigma_{j}}^{t}\left|z_{j}(s)\right| \mathrm{d} s\right]-\frac{\rho_{0}}{2} \exp \left\{-\rho_{0} t\right\} \frac{1}{h_{i}}\left(k_{i}(t)-k^{*}\right)^{2}-k^{*} z_{i}^{2}(t)
\end{aligned}
$$




$$
\begin{aligned}
\leq & \frac{1}{2}\left(-2 C_{i}+\sum_{j=1}^{n} F_{j}\left|a_{i j}\right|+\sum_{j=1}^{n} G_{j}\left|b_{i j}\right|+\sum_{j=1}^{n} \sigma_{j} H_{j}\left|d_{i j}\right|-2 k^{*}\right) z_{i}^{2}(t)-\rho_{0} \Upsilon_{i}(t) \\
& +\frac{1}{2} \sum_{j=1}^{n} F_{j}\left|a_{i j}\right| z_{j}^{2}(t)+\frac{1}{2} \sum_{j=1}^{n} G_{j}\left|b_{i j}\right| z_{j}^{2}\left(t-\tau_{j}\right)+\frac{1}{2} \sum_{j=1}^{n} H_{j}\left|d_{i j}\right| \int_{t-\sigma_{j}}^{t} z_{j}^{2}(s) \mathrm{d} s \\
= & \left(\alpha_{i}-2 k^{*}\right) P_{i}(t)-\rho_{0} \Upsilon_{i}(t)+\sum_{j=1, j \neq i}^{n} F_{j}\left|a_{i j}\right| P_{j}(t)+\sum_{j=1}^{n} G_{j}\left|b_{i j}\right| P_{j}\left(t-\tau_{j}\right) \\
& +\sum_{j=1}^{n} H_{j}\left|d_{i j}\right| \int_{0}^{\sigma_{j}} P_{j}(t-s) \mathrm{d} s, \quad i \in \mathfrak{T} .
\end{aligned}
$$

Choose $k^{*} \geq\left(\alpha_{\kappa}+\rho_{0}\right) / 2$ for all $\kappa \in \mathfrak{T}$, then it follows from (11) that when $\overline{\mathrm{T}}_{l-1} \leq t \leq$ $\overline{\mathrm{T}}_{l-1}+\theta_{l} \mathrm{~T}_{l}, l \in \mathbb{N}^{+}$,

$$
\begin{aligned}
\dot{V}_{i}(t) \leq & -\rho_{0} V_{i}(t)+\sum_{j=1, j \neq i}^{n} F_{j}\left|a_{i j}\right| V_{j}(t)+\sum_{j=1}^{n} G_{j}\left|b_{i j}\right| V_{j}\left(t-\tau_{j}\right) \\
& +\sum_{j=1}^{n} H_{j}\left|d_{i j}\right| \int_{0}^{\sigma_{j}} V_{j}(t-s) \mathrm{d} s, \quad i \in \mathfrak{T} .
\end{aligned}
$$

Similarly, when $\overline{\mathrm{T}}_{l-1}+\theta_{l} \mathrm{~T}_{l}<t<\overline{\mathrm{T}}_{l}, l \in \mathbb{N}^{+}$, we can obtain

$$
\begin{aligned}
\dot{V}_{i}(t)= & z_{i}(t)\left[-\tilde{c}_{i}\left(z_{i}(t)\right)+\sum_{j=1}^{n} a_{i j} \tilde{f}_{j}\left(z_{j}(t)\right)+\sum_{j=1}^{n} b_{i j} \tilde{g}_{j}\left(z_{j}\left(t-\tau_{j}\right)\right)\right. \\
& \left.+\sum_{j=1}^{n} d_{i j} \int_{t-\sigma_{j}}^{t} \tilde{h}_{j}\left(z_{j}(s)\right) \mathrm{d} s\right]-\frac{\rho_{0}}{2} \exp \left\{-\rho_{0} t\right\} \frac{1}{h_{i}}\left(k_{i}\left(\overline{\mathrm{T}}_{l-1}+\theta_{l} \mathrm{~T}_{l}\right)-k^{*}\right)^{2} \\
\leq & \left(\alpha_{i}-\alpha^{+}\right) P_{i}(t)+\alpha^{+} P_{i}(t)+\sum_{j=1, j \neq i}^{n} F_{j}\left|a_{i j}\right| P_{j}(t)+\sum_{j=1}^{n} G_{j}\left|b_{i j}\right| P_{j}\left(t-\tau_{j}\right) \\
& +\sum_{j=1}^{n} H_{j}\left|d_{i j}\right| \int_{0}^{\sigma_{j}} P_{j}(t-s) \mathrm{d} s \\
\leq & \alpha^{+} V_{i}(t)+\sum_{j=1, j \neq i}^{n} F_{j}\left|a_{i j}\right| V_{j}(t)+\sum_{j=1}^{n} G_{j}\left|b_{i j}\right| V_{j}\left(t-\tau_{j}\right) \\
& +\sum_{j=1}^{n} H_{j}\left|d_{i j}\right| \int_{0}^{\sigma_{j}} V_{j}(t-s) \mathrm{d} s, \quad i \in \mathfrak{T} .
\end{aligned}
$$

Take $M_{0}=\sup _{-\eta \leq v \leq 0}\left\{\max _{i \in \mathfrak{T}} V_{i}(v)\right\}, Q_{i}(t)=\exp \{\lambda t\} V_{i}(t)$, where $t \geq-\eta$ and $i \in \mathfrak{T}$. Let $W_{i}(t)=Q_{i}(t)-h M_{0}$, where $h>1$ is a constant and $i \in \mathfrak{T}$. Then it is easy to see that

$$
W_{i}(t)<0, \quad \text { for all } t \in\left[-\eta, \overline{\mathrm{T}}_{0}\right] \text { and } i \in \mathfrak{T} \text {. }
$$

Next, we prove that

$$
W_{i}(t)<0, \quad \text { for all } t \in\left[\overline{\mathrm{T}}_{0}, \theta_{1} \mathrm{~T}_{1}\right] \text { and } i \in \mathfrak{T} \text {. }
$$


Otherwise, by (15), there exist $\ell \in \mathfrak{T}$ and $t^{*} \in\left(\overline{\mathrm{T}}_{0}, \theta_{1} \mathrm{~T}_{1}\right]$ such that

$$
W_{\ell}\left(t^{*}\right)=0, \quad \dot{W}_{\ell}\left(t^{*}\right) \geq 0, \quad W_{j}\left(t^{*}\right) \leq 0, \quad \forall j \in \mathfrak{T} \backslash\{\ell\},
$$

and for all $i \in \mathfrak{T}$

$$
W_{i}(t)<0, \quad-\eta \leq t<t^{*}
$$

According to (12), we have

$$
\begin{aligned}
\dot{W}_{\ell}\left(t^{*}\right)= & \lambda Q_{\ell}\left(t^{*}\right)+\exp \left\{\lambda t^{*}\right\} \dot{V}_{\ell}\left(t^{*}\right) \\
\leq & \lambda Q_{\ell}\left(t^{*}\right)-\rho_{0} Q_{\ell}\left(t^{*}\right)+\sum_{j=1, j \neq \ell}^{n} F_{j}\left|a_{\ell j}\right| \exp \left\{\lambda t^{*}\right\} V_{j}\left(t^{*}\right) \\
& +\sum_{j=1}^{n} G_{j}\left|b_{\ell j}\right| \exp \left\{\lambda t^{*}\right\} V_{j}\left(t^{*}-\tau_{j}\right) \\
& +\sum_{j=1}^{n} H_{j}\left|d_{\ell j}\right| \int_{0}^{\sigma_{j}} \exp \left\{\lambda t^{*}\right\} V_{j}\left(t^{*}-s\right) \mathrm{d} s .
\end{aligned}
$$

Based on (16) and (17), we can obtain

$$
V_{\ell}\left(t^{*}\right)=h M_{0} \exp \left\{-\lambda t^{*}\right\}, \quad V_{j}\left(t^{*}\right) \leq h M_{0} \exp \left\{-\lambda t^{*}\right\}, \quad \forall j \in \mathfrak{T} \backslash\{\ell\},
$$

and for all $j \in \mathfrak{T}$

$$
V_{j}(t)<h M_{0} \exp \{-\lambda t\}, \quad-\eta \leq t<t^{*} .
$$

This means that for all $j \in \mathfrak{T}$

$$
\exp \left\{\lambda t^{*}\right\} V_{j}\left(t^{*}-\tau_{j}\right)<\exp \{\lambda \tau\} Q_{\ell}\left(t^{*}\right)
$$

and

$$
\exp \left\{\lambda t^{*}\right\}\left(\sup _{t *-\sigma \leq v \leq t^{*}} V_{j}(v)\right) \leq \exp \{\lambda \sigma\} Q_{\ell}\left(t^{*}\right) .
$$

Then, using (8), we get

$$
\dot{W}_{\ell}\left(t^{*}\right)<\left(\lambda-\rho_{0}+\beta_{\ell}+\exp \{\lambda \tau\} \gamma_{\ell}+\exp \{\lambda \sigma\} \zeta_{\ell}\right) Q_{\ell}\left(t^{*}\right) \leq 0
$$

This contradicts the second inequality in (16), which indicates that (15) holds. Combining with (14), we can obtain

$$
V_{i}(t)<h M_{0} \exp \{-\lambda t\}, \quad \text { for all } t \in\left[-\eta, \theta_{1} \mathrm{~T}_{1}\right] \text { and } i \in \mathfrak{T} \text {. }
$$

Let $\varpi=\rho_{0}+\alpha^{+}$. Now, we prove that

$$
\Omega_{i}(t)=Q_{i}(t)-h M_{0} \exp \left\{\varpi\left(t-\theta_{1} \mathrm{~T}_{1}\right)\right\}<0, \quad \text { for all } t \in\left(\theta_{1} \mathrm{~T}_{1}, \overline{\mathrm{T}}_{1}\right) \text { and } i \in \mathfrak{T} \text {. }
$$


Otherwise, there exist $\ell \in \mathfrak{T}$ and $t^{* *} \in\left(\theta_{1} \mathrm{~T}_{1}, \widetilde{\mathrm{T}}_{1}\right)$ such that

$$
\Omega_{\ell}\left(t^{* *}\right)=0, \quad \dot{\Omega}_{\ell}\left(t^{* *}\right) \geq 0, \quad \Omega_{j}\left(t^{* *}\right) \leq 0, \quad \forall j \in \mathfrak{T} \backslash\{\ell\}
$$

and for all $i \in \mathfrak{T}$

$$
\Omega_{i}(t)<0, \quad \theta_{1} \mathrm{~T}_{1} \leq t<t^{* *}
$$

For $j \in \mathfrak{T}$, if $\theta_{1} \mathrm{~T}_{1} \leq t^{* *}-\tau_{j}<t^{* *}$, it follows from (22) and (23) that

$$
\exp \left\{\lambda t^{* *}\right\} V_{j}\left(t^{* *}-\tau_{j}\right)<\exp \{\lambda \tau\} h M_{0} \exp \left\{\varpi\left(t^{* *}-\theta_{1} \mathrm{~T}_{1}\right)\right\}=\exp \{\lambda \tau\} Q_{\ell}\left(t^{* *}\right),
$$

and if $-\eta \leq t^{* *}-\tau_{j}<\theta_{1} \mathrm{~T}_{1}$, it follows from (20) and (22) that

$$
\exp \left\{\lambda t^{* *}\right\} V_{j}\left(t^{* *}-\tau_{j}\right)<\exp \{\lambda \tau\} h M_{0} \leq \exp \{\lambda \tau\} Q_{\ell}\left(t^{* *}\right)
$$

Therefore, for any $j \in \mathfrak{T}$, one always has

$$
\exp \left\{\lambda t^{* *}\right\} V_{j}\left(t^{* *}-\tau_{j}\right)<\exp \{\lambda \tau\} Q_{\ell}\left(t^{* *}\right)
$$

Similarly, with the same analysis, we get

$$
\exp \left\{\lambda t^{* *}\right\}\left(\sup _{t^{* *}-\sigma \leq v \leq t^{* *}} V_{j}(v)\right) \leq \exp \{\lambda \sigma\} Q_{\ell}\left(t^{* *}\right), \quad \forall j \in \mathfrak{T} .
$$

Then it follows from (8) and (13) that

$$
\begin{aligned}
\dot{\Omega}_{\ell}\left(t^{* *}\right)= & \lambda Q_{\ell}\left(t^{* *}\right)+\exp \left\{\lambda t^{* *}\right\} \dot{V}_{\ell}\left(t^{* *}\right)-\varpi h M_{0} \exp \left\{\varpi\left(t^{* *}-\theta_{1} \mathrm{~T}_{1}\right)\right\} \\
\leq & \left(\lambda+\alpha^{+}-\varpi\right) Q_{\ell}\left(t^{* *}\right)+\sum_{j=1, j \neq \ell}^{n} F_{j}\left|a_{\ell j}\right| \exp \left\{\lambda t^{* *}\right\} V_{j}\left(t^{* *}\right) \\
& +\sum_{j=1}^{n} G_{j}\left|b_{\ell j}\right| \exp \left\{\lambda t^{* *}\right\} V_{j}\left(t^{* *}-\tau_{j}\right)+\sum_{j=1}^{n} H_{j}\left|d_{\ell j}\right| \int_{0}^{\sigma_{j}} \exp \left\{\lambda t^{* *}\right\} V_{j}\left(t^{* *}-s\right) \mathrm{d} s \\
< & \left(\lambda-\rho_{0}+\beta_{\ell}+\exp \{\lambda \tau\} \gamma_{\ell}+\exp \{\lambda \sigma\} \zeta_{\ell}\right) Q_{\ell}\left(t^{* *}\right) \leq 0,
\end{aligned}
$$

which contradicts the second inequality in (22), i.e., (21) holds. Therefore,

$$
\begin{aligned}
Q_{i}(t) & <h M_{0} \exp \left\{\varpi\left(t-\theta_{1} \mathrm{~T}_{1}\right)\right\} \\
& \leq h M_{0} \exp \left\{\varpi\left(1-\theta_{1}\right) \mathrm{T}_{1}\right\}, \quad \text { for all } t \in\left(\theta_{1} \mathrm{~T}_{1}, \overline{\mathrm{T}}_{1}\right) \text { and } i \in \mathfrak{T} .
\end{aligned}
$$

Combining with inequalities (14) and (15), we obtain

$$
Q_{i}(t)<h M_{0} \exp \left\{\varpi\left(1-\theta_{1}\right) \mathrm{T}_{1}\right\}, \quad \text { for all } t \in\left[-\eta, \overline{\mathrm{T}}_{1}\right) \text { and } i \in \mathfrak{T} \text {. }
$$

Similar to the proofs of (15) and (21), we can prove that

$$
Q_{i}(t)<h M_{0} \exp \left\{\varpi\left(1-\theta_{1}\right) \mathrm{T}_{1}\right\}, \quad \text { for all } t \in\left[\overline{\mathrm{T}}_{1}, \overline{\mathrm{T}}_{1}+\theta_{2} \mathrm{~T}_{2}\right] \text { and } i \in \mathfrak{T} \text {, }
$$


and

$$
\begin{aligned}
Q_{i}(t) & <h M_{0} \exp \left\{\varpi\left(1-\theta_{1}\right) \mathrm{T}_{1}\right\} \exp \left\{\varpi\left(t-\overline{\mathrm{T}}_{1}-\theta_{2} \mathrm{~T}_{2}\right)\right\} \\
& =h M_{0} \exp \left\{\varpi\left(t-\left(\theta_{1} \mathrm{~T}_{1}+\theta_{2} \mathrm{~T}_{2}\right)\right)\right\} \\
& \leq h M_{0} \exp \left\{\varpi\left(\left(1-\theta_{1}\right) \mathrm{T}_{1}+\left(1-\theta_{2}\right) \mathrm{T}_{2}\right)\right\},
\end{aligned}
$$

for all $t \in\left(\overline{\mathrm{T}}_{1}+\theta_{2} \mathrm{~T}_{2}, \overline{\mathrm{T}}_{2}\right)$ and $i \in \mathfrak{T}$.

Denote $\theta_{0}=0$. Since $\varpi>0$, by mathematical induction, then we can derive the following estimation of $Q_{\kappa}(t)$ for any positive integer $l$ and $\kappa \in \mathfrak{T}$.

For all $\overline{\mathrm{T}}_{l-1} \leq t \leq \overline{\mathrm{T}}_{l-1}+\theta_{l} \mathrm{~T}_{l}, l \in \mathbb{N}^{+}$and $\kappa \in \mathfrak{T}$

$$
\begin{aligned}
Q_{\kappa}(t) & <h M_{0} \exp \left\{\varpi\left(\sum_{j=0}^{l-1}\left(1-\theta_{j}\right) \mathrm{T}_{j}\right)\right\} \\
& \leq h M_{0} \exp \left\{\varpi\left(1-\theta_{\text {inf }}\right)\left(\sum_{j=0}^{l-1} \mathrm{~T}_{j}\right)\right\} \leq h M_{0} \exp \left\{\varpi\left(1-\theta_{\text {inf }}\right) t\right\} .
\end{aligned}
$$

And for all $\overline{\mathrm{T}}_{l-1}+\theta_{l} \mathrm{~T}_{l}<t<\overline{\mathrm{T}}_{l}, l \in \mathbb{N}^{+}$and $\kappa \in \mathfrak{T}$

$$
\begin{aligned}
Q_{\kappa}(t) & <h M_{0} \exp \left\{\varpi\left(t-\left(\sum_{j=0}^{l} \theta_{j} \mathrm{~T}_{j}\right)\right)\right\} \\
& \leq h M_{0} \exp \left\{\varpi t-\varpi \theta_{\text {inf }}\left(\sum_{j=0}^{l} \mathrm{~T}_{j}\right)\right\}<h M_{0} \exp \left\{\varpi\left(1-\theta_{\text {inf }}\right) t\right\} .
\end{aligned}
$$

Noting that $Q_{\kappa}(t)=\exp \{\lambda t\} V_{\kappa}(t), \kappa \in \mathfrak{T}$ and $\varpi=\rho_{0}+a^{+}$, it follows from (24) and (25) that

$$
\begin{aligned}
V_{\kappa}(t) & <h M_{0} \exp \{-\lambda t\} \exp \left\{\varpi\left(1-\theta_{\mathrm{inf}}\right) t\right\} \\
& =h M_{0} \exp \left\{-\left(\lambda-\left(\rho_{0}+a^{+}\right)\left(1-\theta_{\mathrm{inf}}\right)\right) t\right\}
\end{aligned}
$$

for all $\kappa \in \mathfrak{T}$ and $t \geq 0$.

Let $h \rightarrow 1$, from the definition of $V_{i}(t)$, we have

$$
\left|z_{i}(t)\right| \leq \sqrt{2 M_{0}} \exp \left\{-\frac{1}{2}\left[\lambda-\left(\rho_{0}+a^{+}\right)\left(1-\theta_{\text {inf }}\right)\right] t\right\}
$$

for all $i \in \mathfrak{T}$ and $t \geq 0$, which implies that

$$
\|y(t)-x(t-\omega)\| \leq \sqrt{2 M_{0}} \exp \left\{-\frac{1}{2}\left[\lambda-\left(\rho_{0}+a^{+}\right)\left(1-\theta_{\text {inf }}\right)\right] t\right\}, \quad t \geq 0
$$

According to condition (7), we can conclude that, by the adaptive nonperiodical intermittent controllers (3)-(5), the globally exponential lag synchronization between the driveresponse systems (1) and (2) can be achieved. The proof is thus completed. 
In the case that both the control periods and the control widths are fixed, i.e., $t_{l+1}-t_{l} \equiv \mathrm{T}$ and $\delta_{l} \equiv \delta$, for all $l \in \mathbb{N}^{+}$, where $\mathrm{T}$ and $\delta$ are two positive constants, then the control type becomes the adaptive periodically intermittent control, which has been investigated in [39-41]. Denote $\theta=\delta / T$, then the following result can easily be obtained from Theorem 1 .

Corollary 1 Suppose that Assumptions 1-2 hold, by the adaptive periodically intermittent control, the globally exponential lag synchronization between the drive-response systems (1) and (2) can be achieved if there exists a positive constant $\rho_{0}>\pi_{0}$ such that

$$
1-\frac{\lambda}{\rho_{0}+\alpha^{+}}<\theta<1
$$

where $\alpha^{+}$and $\lambda$ are the same as those in Theorem 1.

If there is no distributed delay in system (1), i.e., $\sigma_{j} \equiv 0$ for all $j \in \mathfrak{T}$, then system (1) turns into the following neural networks with discrete delays:

$$
\dot{x}_{i}(t)=-c_{i}\left(x_{i}(t)\right)+\sum_{j=1}^{n} a_{i j} f_{j}\left(x_{j}(t)\right)+\sum_{j=1}^{n} b_{i j} g_{j}\left(x_{j}\left(t-\tau_{j}\right)\right)+I_{i}, \quad i \in \mathfrak{T} .
$$

The corresponding controlled response network is described by

$$
\dot{y}_{i}(t)=-c_{i}\left(y_{i}(t)\right)+\sum_{j=1}^{n} a_{i j} f_{j}\left(y_{j}(t)\right)+\sum_{j=1}^{n} b_{i j} g_{j}\left(y_{j}\left(t-\tau_{j}\right)\right)+I_{i}+u_{i}(t), \quad i \in \mathfrak{T},
$$

where $u_{i}(t)$ is the same as equation (3). In this case, $\zeta_{\kappa} \equiv 0$ for all $\kappa \in \mathfrak{T}$, and so $\pi_{0}=$ $\max _{\kappa \in \mathfrak{T}}\left\{\beta_{\kappa}+\gamma_{\kappa}\right\}$. Consequently, we can derive the following results from Theorem 1 and Corollary 1 .

Corollary 2 Suppose that Assumptions 1-2 hold and $\inf _{l \in \mathbb{N}^{+}}\left\{\theta_{l}\right\}=\theta_{\text {inf }}>0$, by the adaptive nonperiodical intermittent controllers (3)-(5), the globally exponential lag synchronization between the drive-response systems (30) and (31) can be achieved if there exists a positive constant $\rho_{0}>\pi_{0}$ such that

$$
\lambda-\left(1-\theta_{\text {inf }}\right)\left(\rho_{0}+\alpha^{+}\right)>0,
$$

where $\alpha^{+}=\max \{0, \alpha\}, \lambda=\min _{\kappa \in \mathfrak{T}}\left\{\lambda_{\kappa}\right\}$, and $\lambda_{\kappa}$ is the unique positive solution of the equation $\lambda_{\kappa}-\rho_{0}+\beta_{\kappa}+\gamma_{\kappa} \exp \left\{\lambda_{\kappa} \tau\right\}=0$.

Corollary 3 Suppose that Assumptions 1-2 hold, by the adaptive periodically intermittent control, the globally exponential lag synchronization between the drive-response systems (30) and (31) can be achieved if there exists a positive constant $\rho_{0}>\pi_{0}$ such that

$$
1-\frac{\lambda}{\rho_{0}+\alpha^{+}}<\theta<1
$$

where $\alpha^{+}$and $\lambda$ are the same as those in Corollary 2. 
Remark 2 Evidently, the adaptive nonperiodically intermittent control strategy presented in this paper can also be utilized to realize complete synchronization of neural networks with mixed delays, only if let propagation delay $\omega=0$.

Remark 3 When $t_{l+1}-t_{l} \equiv \delta_{l}$ or $\theta_{l} \equiv 1$, for all $l \in \mathbb{N}^{+}$, the adaptive intermittent control turns into the continuous-time adaptive control. In this case, the condition $\rho_{0}>\pi_{0}$ ensure the globally exponential lag synchronization between the drive-response systems (1) and (2) can be achieved under the continuous-time adaptive control, because condition (7) holds automatically.

Remark 4 In [13], the authors studied the exponential lag synchronization problem for neural networks with mixed delays via intermittent control. However, the designed intermittent controller in [13] is periodic, which requires both the control period and the control width should be fixed. This requirement is obviously unreasonable and unnecessary in reality. In this paper, by proposing an adaptive intermittent control scheme, the exponential lag synchronization of neural networks with mixed delays was further investigated. Noticeably, here the adaptive intermittent controller is nonperiodic, it possesses different control periods as well as different control widths. Hence, our control strategy is more feasible than that in [13].

Remark 5 In $[40,41,50]$, under adaptive intermittent control, the complete synchronization and cluster synchronization of directed dynamical networks were studied by constructing a piecewise Lyapunov function. However, the piecewise Lyapunov function given in $[40,41,50]$ isn't continuous at $t=t_{l+1}, l \in \mathbb{N}^{+}$, and therefore only some criteria ensuring globally asymptotical synchronization were derived in $[40,41,50]$. In this paper, we introduce some piecewise continuous Lyapunov functions (see equation (10)), and then by means of which and piecewise analytic method, we derive some sufficient conditions for globally exponential lag synchronization between two delayed neural networks (1) and (2) under the adaptive intermittent control. Therefore, the results established here extend those in $[40,41,50]$.

Remark 6 It should be stressed that conditions (7) and (32) show that the lag synchronization criteria are related with the quantity $\theta_{\text {inf }}$ rather than the control periods $\mathrm{T}_{l}\left(l \in \mathbb{N}^{+}\right)$ or the control widths $\delta_{l}\left(l \in \mathbb{N}^{+}\right)$. Therefore, for achieving the lag synchronization, one can arbitrarily select the control periods $\mathrm{T}_{l}, l \in \mathbb{N}^{+}$. Specially, for practical problems, the control periods $\mathrm{T}_{l}, l \in \mathbb{N}^{+}$can be chosen in the light of practical requirements.

Remark 7 For illuminating how to design suitable adaptive nonperiodical intermittent controllers to make the lag synchronization between the drive and response systems (1) and (2) can be achieved, the following steps are given:

(1) Compute the values of $\tau, \sigma, \alpha_{\kappa}, \beta_{\kappa}, \gamma_{\kappa}$ and $\zeta_{\kappa}, \kappa \in \mathfrak{T}$ according to their definitions, and then figure out the values of $\pi_{0}, \alpha$, and $\alpha^{+}$.

(2) Select a $\rho_{0}$ satisfying $\rho_{0}>\pi_{0}$, and then calculate $\lambda_{i}$ and $\lambda$ via mathematical software MATLAB.

(3) By means of condition (7), compute a bound of the quantity $\theta_{\text {inf }}$ satisfying $1-\lambda /\left(\rho_{0}+\alpha^{+}\right)<\theta_{\text {inf }}<1$, and then arbitrarily select the control rates $\theta_{l}, l \in \mathbb{N}^{+}$such that the inequality holds. 
(4) Choose randomly the control periods $\mathrm{T}_{l}, l \in \mathbb{N}^{+}$and propagation delay $\omega$.

(5) Based on the above chosen $\rho_{0}, \theta_{l}, \mathrm{~T}_{l}, l \in \mathbb{N}^{+}$, and $\omega$, design the adaptive intermittent controllers given by (3)-(5).

\section{Numerical examples}

In this section, we give a numerical example to illustrate the effectiveness of the derived theoretical results.

Consider the neural networks with mixed delays described by

$$
\begin{aligned}
\dot{x}_{i}(t)= & -c_{i} x_{i}(t)+\sum_{j=1}^{2} a_{i j} f_{j}\left(x_{j}(t)\right)+\sum_{j=1}^{2} b_{i j} g_{j}\left(x_{j}(t-1)\right) \\
& +\sum_{j=1}^{2} d_{i j} \int_{t-0.8}^{t} h_{j}\left(x_{j}(s)\right) \mathrm{d} s, \quad i \in \mathfrak{T}=\{1,2\},
\end{aligned}
$$

where $f_{j}(x)=g_{j}(x)=h_{j}(x)=\tanh (x)$, and $c_{1}=c_{2}=1, a_{11}=1.3, a_{12}=-0.1, a_{21}=-1.0, a_{22}=$ $0.2, b_{11}=-1.5, b_{12}=-0.4, b_{21}=0.1, b_{22}=-2, d_{11}=-0.4, d_{12}=0.1, d_{21}=0.0, d_{22}=-0.5$. The numerical simulation of system (34) is shown in Figure 1, which indicates that system (34) has a chaotic attractor. The corresponding response system is of the form

$$
\begin{aligned}
\dot{y}_{i}(t)= & -c_{i} y_{i}(t)+\sum_{j=1}^{2} a_{i j} f_{j}\left(y_{j}(t)\right)+\sum_{j=1}^{2} b_{i j} g_{j}\left(y_{j}(t-1)\right) \\
& +\sum_{j=1}^{2} d_{i j} \int_{t-0.8}^{t} h_{j}\left(y_{j}(s)\right) \mathrm{d} s+u_{i}(t), \quad i \in \mathfrak{T},
\end{aligned}
$$

where the controller $u_{i}(t)$ is described by equations (3)-(5).

By simple calculation, we get $F_{i}=G_{i}=H_{i}=1$ for $i=1,2$, and $\alpha_{1}=3.0, \alpha_{2}=1.9, \beta_{1}=0.1$, $\beta_{2}=1, \gamma_{1}=1.9, \gamma_{2}=2.1, \zeta_{1}=0.4, \zeta_{2}=0.4, \pi_{0}=3.5, \alpha^{+}=\alpha=3.1$. Select $\rho_{0}=10>\pi_{0}$, then

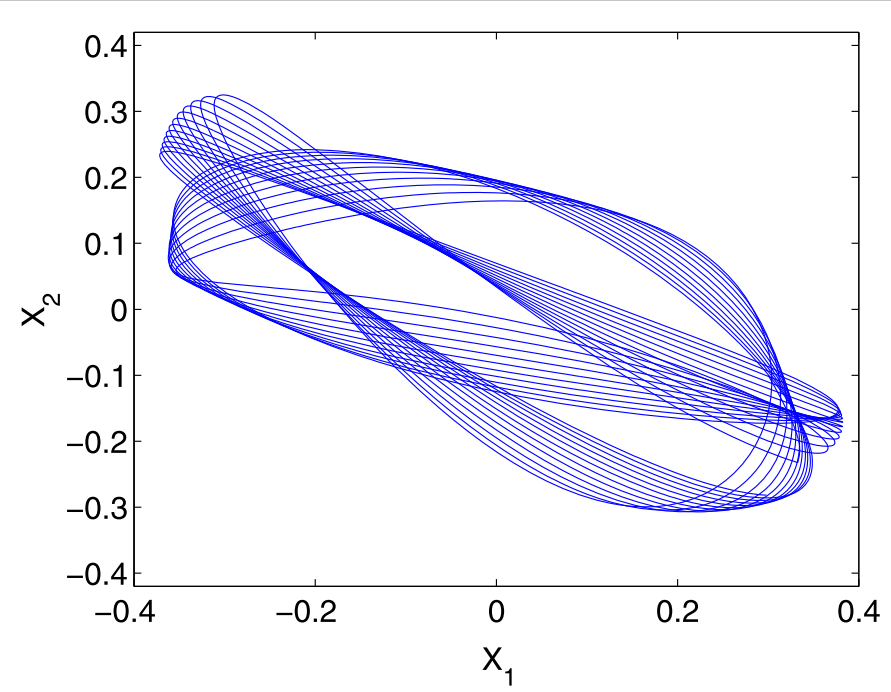

Figure 1 The chaotic attractor of system (34) with initial values $x_{1}(v)=0.3$ and $x_{2}(v)=-0.1$ for $v \in[-1,0]$. 
we have $\lambda_{1}=1.3547, \lambda_{2}=1.1752$, and therefore $\lambda=1.1752$. From condition (7), one can obtain $0.9096<\theta_{\text {inf }}<1$. For brevity, we choose $\theta_{l} \equiv 0.91, \mathrm{~T}_{l}=t_{l+1}-t_{l}=0.25 l, l \in \mathbb{N}^{+}$, then condition (7) holds. Choosing propagation delay $\omega=3$, the time evolutions of synchronous errors between systems (33) and (34) and adaptive intermittent feedback control gains are shown in Figures 2 and 3, respectively, where the initial condition of the simulation are $x_{1}(v)=0.3, x_{2}(v)=-0.1, y_{1}(v)=0.1, y_{2}(v)=-0.3$ for $v \in[-1,0]$, and $k_{1}(0)=k_{2}(0)=$ $0.2, h_{1}=h_{2}=0.02$. In addition, the lag synchronization between systems (33) and (34) is plotted in Figures 4 and 5 . The numerical example indicates the effectiveness of our control method.



Figure 2 Time evolutions of synchronous errors $e_{1}(t)$ and $e_{2}(t)$ between systems (34) and (35).

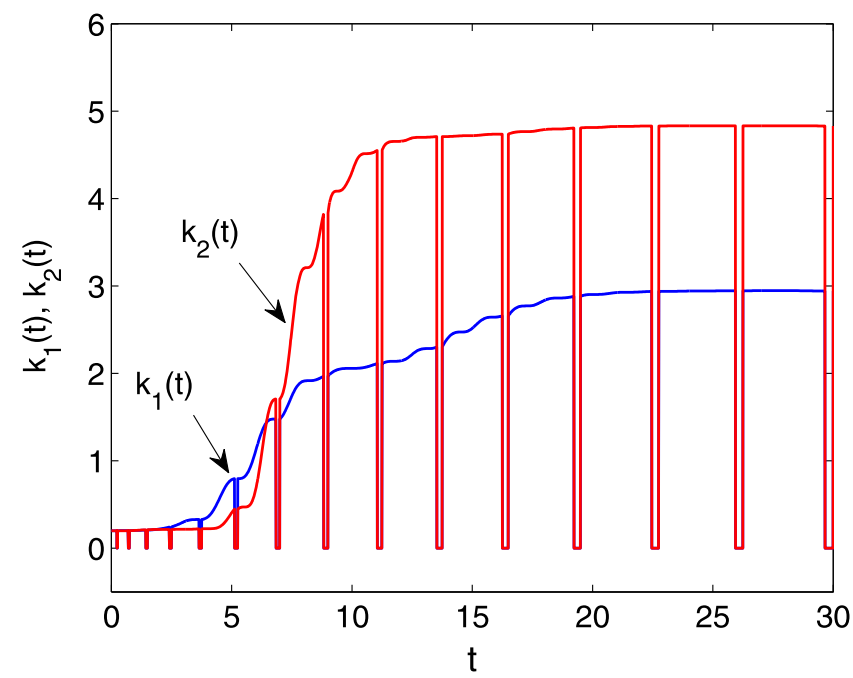

Figure 3 Time evolutions of adaptive intermittent feedback control gains $k_{1}(t)$ and $k_{2}(t)$. 


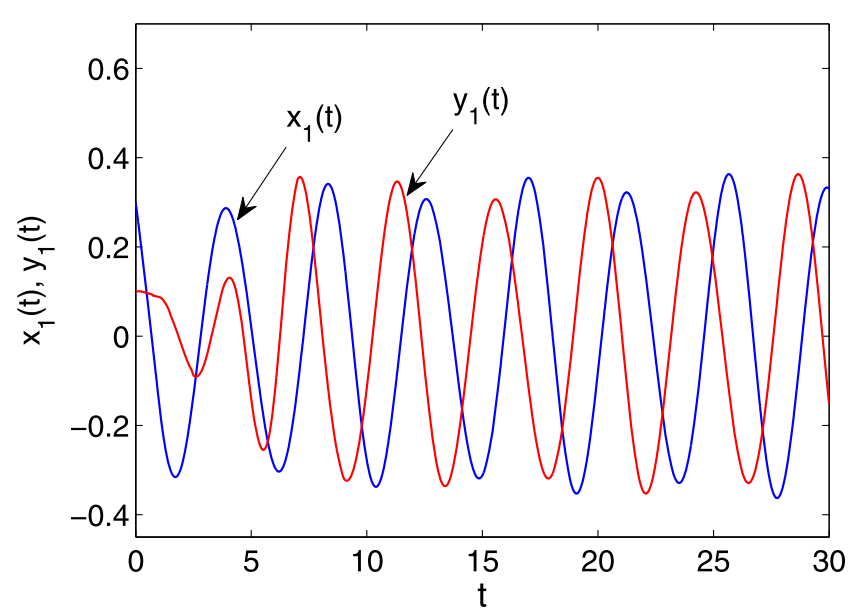

Figure 4 Lag synchronization of $x_{1}(t)$ and $y_{1}(t)$ with propagation delay $\omega=3$.



Figure 5 Lag synchronization of $x_{2}(t)$ and $y_{2}(t)$ with propagation delay $\omega=3$.

\section{Conclusions}

In this paper, an adaptive nonperiodically intermittent control scheme is proposed to study the globally exponential lag synchronization problem for a class of neural networks with both discrete and distributed delays. Some criteria ensuring the globally exponential lag synchronization are derived through introducing a piecewise continuous auxiliary function and utilizing piecewise analytic method. Different from previous works, the developed adaptive intermittent control can be nonperiodic, which extends the intermittent control strategy's application scopes. Numerical simulations are also given to verify the feasibility of the obtained theoretical results. It should be noted that the adaptive update law for intermittent feedback control gain designed in this paper explicitly depends on time $t$, which will make the adaptive intermittent control technique not easy to implement in practical applications. Therefore, how to design more reasonable adaptive update rules is an interesting topic to be investigated in the future. 


\section{Acknowledgements}

This work was supported by the National Science Foundation of China (Grant Nos. 11402100 and 11572278) and Young Core Teachers Training Project of Jiangsu University. The authors are grateful to the editor and reviewers for their valuable suggestions and comments.

\section{Competing interests}

The authors declare that they have no competing interests.

\section{Authors' contributions}

All the authors contributed equally to this work. All authors read and approved the final manuscript.

\section{Publisher's Note}

Springer Nature remains neutral with regard to jurisdictional claims in published maps and institutional affiliations.

Received: 21 September 2017 Accepted: 18 January 2018 Published online: 25 January 2018

\section{References}

1. Pecora, LM, Carroll, TL: Synchronization in chaotic systems. Phys. Rev. Lett. 64, 821-824 (1990)

2. Pecora, LM, Carroll, TL, Johnson, GA, Mar, DJ, Heagy, JF: Fundamentals of synchronization in chaotic systems, concepts, and applications. Chaos 7, 520-543 (1997)

3. Boccaletti, S, Kurths, J, Osipov, G, Valladares, DL, Zhou, CS: The synchronization of chaotic systems. Phys. Rep. 366, 1-101 (2002)

4. Kocarev, L, Parlitz, U: Generalized synchronization, predictability, and equivalence of unidirectionally coupled dynamical systems. Phys. Rev. Lett. 76, 1816-1819 (1996)

5. Rosenblum, MG, Pikovsky, AS, Kurths, J: Phase synchronization of chaotic oscillators. Phys. Rev. Lett. 76, 1804-1807 (1996)

6. Rosenblum, MG, Pikovsky, AS, Kurths, J: From phase to lag synchronization in coupled chaotic oscillators. Phys. Rev. Lett. 78, 4193-4196 (1997)

7. Mainieri, R, Rehacek, J: Projective synchronization in three-dimensional chaotic systems. Phys. Rev. Lett. 82, 3042-3045 (1999)

8. Taherion, S, Lai, YC: Observability of lag synchronization of coupled chaotic oscillators. Phys. Rev. E 59, R6247-R6250 (1999)

9. Shahverdiev, EM, Sivaprakasam, S, Shore, KA: Lag times and parameter mismatches in synchronization of unidirectionally coupled chaotic external cavity semiconductor lasers. Phys. Rev. E 66, 037202 (2002)

10. Liu, H, Sun, W, Al-mahbashi, G: Parameter identification based on lag synchronization via hybrid feedback control in uncertain drive-response dynamical networks. Adv. Differ. Equ. 2017, 122 (2017)

11. Li, N, Cao, J, Alsaedi, A, Alsaadi, F: Lag synchronization criteria for memristor-based coupled neural networks via parameter mismatches analysis approach. Neural Comput. 29, 1721-1744 (2017)

12. Huang, J, Li, C, Huang, T, Han, Q: Lag quasisynchronization of coupled delayed systems with parameter mismatch by periodically intermittent control. Nonlinear Dyn. 71, 469-478 (2013)

13. $\mathrm{Hu}, \mathrm{C}, \mathrm{Yu}$, J, Jiang, H, Teng, Z: Exponential lag synchronization for neural networks with mixed delays via periodically intermittent control. Chaos 20, 023108 (2010)

14. Wang, $\mathrm{Q}, \mathrm{Lu}, \mathrm{Q}$, Duan, Z: Adaptive lag synchronization in coupled chaotic systems with unidirectional delay feedback. Int. J. Non-Linear Mech. 45, 640-646 (2010)

15. Wang, Z, Shi, X: Lag synchronization of multiple identical Hindmarsh-Rose neuron models coupled in a ring structure. Nonlinear Dyn. 60, 375-383 (2010)

16. Yang, Y, Cao, J: Exponential synchronization of the complex dynamical networks with a coupling delay and impulsive effects. Nonlinear Anal. 11, 1650-1659 (2010)

17. Bao, H, Park, JH, Cao, J: Exponential synchronization of coupled stochastic memristor-based neural networks with time-varying probabilistic delay coupling and impulsive delay. IEEE Trans. Neural Netw. Learn. Syst. 27, 190-201 (2016)

18. Cai, S, Li, X, Jia, Q, Liu, Z: Exponential cluster synchronization of hybrid-coupled impulsive delayed dynamical networks: average impulsive interval approach. Nonlinear Dyn. 85, 2405-2423 (2016)

19. Li, C, Liao, X, Wong, K: Chaotic lag synchronization of coupled time-delayed systems and its applications in secure communication. Physica D 194, 187-202 (2004)

20. Li, C, Liao, X: Complete and lag synchronization of hyperchaotic systems using small impulses. Chaos Solitons Fractals 22, 857-867 (2004)

21. Yang, X, Cao, J, Long, Y, Rui, W: Adaptive lag synchronization for competitive neural networks with mixed delays and uncertain hybrid perturbations. IEEE Trans. Neural Netw. 21, 1656-1667 (2010)

22. He, W, Qian, F, Han, QL, Cao, J: Lag quasi-synchronization of coupled delayed systems with parameter mismatch. IEEE Trans. Circuits Syst. I 58, 1345-1357 (2011)

23. Liu, Q, Zhang, S: Adaptive lag synchronization of chaotic Cohen-Grossberg neural networks with discrete delays. Chaos 22, $033123(2012)$

24. Lu, J, Ho, DWC: Globally exponential synchronization and synchronizability for general dynamical networks. IEEE Trans. Syst. Man Cybern., Part B, Cybern. 40, 350-361 (2010)

25. Lu, J, Wang, Z, Cao, J, Ho, DWC, Kurths, J: Pinning impulsive stabilization of nonlinear dynamical networks with time-varying delay. Int. J. Bifurc. Chaos Appl. Sci. Eng. 22, 1250176 (2012)

26. Lu, J, Ding, C, Lou, J, Cao, J: Outer synchronization of partially coupled dynamical networks via pinning impulsive controllers. J. Franklin Inst. 352, 5024-5041 (2015)

27. Li, Y: Impulsive synchronization of stochastic neural networks via controlling partial states. Neural Process. Lett. 46, 59-69 (2017) 
28. Huang, T, Li, C, Liu, X: Synchronization of chaotic systems with delay using intermittent linear state feedback. Chaos $18,033122(2008)$

29. Huang, T, Li, C, Yu, W, Chen, G: Synchronization of delayed chaotic systems with parameter mismatches by using intermittent linear state feedback. Nonlinearity 22, 569-584 (2009)

30. Hu, C, Yu, J, Jiang, H, Teng, Z: Exponential stabilization and synchronization of neural networks with time-varying delays via periodically intermittent control. Nonlinearity 23, 2369-2391 (2010)

31. Cai, S, Hao, J, Liu, Z: Exponential synchronization of chaotic systems with time-varying delays and parameter mismatches via intermittent control. Chaos 21, 023112 (2011)

32. Cai, S, Hao, J, He, Q, Liu, Z: New results on synchronization of chaotic systems with time-varying delays via intermittent control. Nonlinear Dyn. 67, 393-402 (2012)

33. Li, C, Feng, G, Liao, X: Stabilization of nonlinear systems via periodically intermittent control. IEEE Trans. Circuits Syst. II 54, 1019-1023 (2007)

34. Xia, W, Cao, J: Pinning synchronization of delayed dynamical networks via periodically intermittent control. Chaos 19 $013120(2009)$

35. Cai, S, Hao, J, He, Q, Liu, Z: Exponential synchronization of complex delayed dynamical networks via pinning periodically intermittent control. Phys. Lett. A 375, 1965-1971 (2011)

36. Cai, S, Zhou, P, Liu, Z: Pinning synchronization of hybrid-coupled directed delayed dynamical network via intermittent control. Chaos 24, 033102 (2014)

37. $\mathrm{Hu}, \mathrm{C}, \mathrm{Yu}, \mathrm{J}$, Jiang, $\mathrm{H}$, Teng, Z: Exponential synchronization of complex networks with finite distributed delays coupling. IEEE Trans. Neural Netw. 22, 1999-2010 (2011)

38. Liu, $X, C h e n, T$ : Cluster synchronization in directed networks via intermittent pinning control. IEEE Trans. Neural Netw. 22, 1009-1020 (2011)

39. Zhao, M, Zhang, H, Wang, Z, Liang, H: Synchronization between two general complex networks with time-delay by adaptive periodically intermittent pinning control. Neurocomputing 144, $215-221$ (2014)

40. $\mathrm{Hu}, \mathrm{C}$, Jiang, $\mathrm{H}$ : Pinning synchronization for directed networks with node balance via adaptive intermittent control. Nonlinear Dyn. 80, 295-307 (2015)

41. Cai, S, Jia, Q, Liu, Z: Cluster synchronization for directed heterogeneous dynamical networks via decentralized adaptive intermittent pinning control. Nonlinear Dyn. 82, 689-702 (2015)

42. Cai, S, Zhou, P, Liu, Z: Intermittent pinning control for cluster synchronization of delayed heterogeneous dynamical networks. Nonlinear Anal. Hybrid Syst. 18, 134-155 (2015)

43. Mei, J, Jiang, M, Wu, Z, Wang, X: Periodically intermittent controlling for finite-time synchronization of complex dynamical networks. Nonlinear Dyn. 80, 295-307 (2015)

44. Liu, X, Chen, T: Synchronization of linearly coupled networks with delays via aperiodically intermittent pinning control. IEEE Trans. Neural Netw. Learn. Syst. 26, 2396-2407 (2015)

45. Liu, X, Liu, Y, Zhou, L: Quasi-synchronization of nonlinear coupled chaotic systems via aperiodically intermittent pinning control. Neurocomputing 173, 759-767 (2016)

46. Liu, M, Jiang, H, Hu, C: Synchronization of hybrid-coupled delayed dynamical networks via aperiodically intermittent pinning control. J. Franklin Inst. 353, 2722-2742 (2016)

47. Zhang, W, Li, C, Huang, T, Xiao, M: Synchronization of neural networks with stochastic perturbation via aperiodically intermittent control. Neural Netw. 71, 105-111 (2015)

48. Song, Q, Huang, T: Stabilization and synchronization of chaotic systems with mixed time-varying delays via intermittent control with non-fixed both control period and control width. Neurocomputing 154, 61-69 (2015)

49. Cai, S, Lei, X, Liu, Z: Outer synchronziation between two hybrid-coupled delayed dynamical networks via aperiodically adaptive intermittent pinning control. Complexity 21, 593-605 (2016)

50. Lei, X, Cai, S, Jiang, S, Liu, Z: Adaptive outer synchronization between two complex delayed dynamical networks via aperiodically intermittent pinning control. Neurocomputing 222, 26-35 (2017)

51. Zhou, P, Cai, S: Pinning synchronization of complex directed dynamical networks under decentralized adaptive strategy for aperiodically intermittent control. Nonlinear Dyn. 90, 287-299 (2017)

52. Gao, J, Cao, J: Aperiodically intermittent synchronization for switching complex networks dependent on topology structure. Adv. Differ. Equ. 2017, 244 (2017)

53. Zhou, P, Cai, S, Jiang, S, Liu, Z: Exponential cluster synchronization in directed community networks via adaptive nonperiodically intermittent pinning control. Physica A 492, 1267-1280 (2018)

54. Liu, Y, Wang, Z, Liu, X: Global exponential stability of generalized recurrent neural networks with discrete and distributed delays. Neural Netw. 19, 667-675 (2006)

55. Cao, J, Li, L: Cluster synchronization in an array of hybrid coupled neural networks with delay. Neural Netw. 22, 335-342 (2009)

56. Leng, $\mathrm{H}, \mathrm{Wu}, \mathrm{Z}$ : Cluster synchronization of community network with distributed time delays via impulsive control. Chin. Phys. B 25, 110501 (2016) 